\title{
Coloduodenal Fistula: The Role of MDCT on Diagnosing a Rare Entity
}

\author{
Giordano Rafael Tronco Alves ${ }^{1 \star}$, John Neoptolemos ${ }^{2}$, Natália Henz Concatto ${ }^{3}$, Bruno Hochhegger ${ }^{4}$ and Klaus Loureiro \\ Irion $^{5}$ \\ ${ }^{1}$ Post-graduation Program in Medicine (Radiology), Federal University of Rio de Janeiro, Rio de Janeiro, Brazil \\ ${ }^{2}$ Department of Molecular and Clinical Cancer Medicine, University of Liverpool, Liverpool, UK \\ ${ }^{3}$ Radiology Division, University of Caxias do Sul, Caxias do Sul, Brazil \\ ${ }^{4}$ Department of Radiology, Federal University of Health Sciences of Porto Alegre, Porto Alegre, Brazil \\ ${ }^{5}$ Department of Radiology, Liverpool Heart and Chest Hospital, Liverpool, UK \\ *Corresponding author: Giordano R. T. Alves, M.D, Post-graduation Program in Medicine (Radiology) from the Federal University of Rio de Janeiro, Professor \\ Rodolpho Paulo Rocco Street, 255, ZC: 21941-913, Rio de Janeiro, Brazil, Tel: 555599159009; Fax: 555599159009; E-mail: grtalves@gmail.com
}

Received: 30 October, 2014; Accepted: 19 March, 2015; Published: 21 March, 2015

\section{Introduction}

Coloduodenal fistula is a very infrequent complication, which can arise from both benign and malignant diseases. It consists in a pathological communication between the lumen of the colon and duodenum. The onset of signs and symptoms is generally sub-acute, and the majority of the patients will present with a non-specific abdominal pain, diarrhoea, nausea and vomiting [1]. Nevertheless, the clinical presentation may vary significantly - depending on the site of fistulisation - and some cases may never be diagnosed.

When not clinically suspected, cross-sectional imaging studies can be the only tool to suggest the diagnosis and provide information necessary for deciding among therapeutic options [2]. In this article, we report a case of coloduodenal fistula arising from a colonic malignancy, diagnosed by multi-detector computed tomography scan (MDCT). A brief discussion regarding the condition and its diagnostic challenges is presented.

\section{Case Report}

A 60-year-old male, ex-smoker, was admitted complaining of weight loss and tiredness for one year. He also complained of recent onset of abdominal discomfort and diarrhoea. Past medical history was otherwise unremarkable, except for chronic hypertension and type- 2 diabetes mellitus. A palpable mass was noted in the right flank. Normocytic normochromic anaemia was detected on haemogram, suggesting the presence of an underlying chronic disease.

Colonoscopy revealed multiple colonic polyps and a mass in the ascending colon. Adenocarcinoma of the colon was confirmed on histopathology. MDCT imaging (Figure 1, A-axial, B-coronal and $\mathrm{C}$-sagittal views, $\mathrm{C}=$ colon $/ \mathrm{D}=$ duodenum and $\mathrm{GB}=$ gallbladder) shows a mass in the ascending colon, with infiltration of the adjacent fat and duodenal invasion with a large fistulous tract communicating the lumen of these two segments of the bowel loops (indicated by arrows). Liver metastases and enlarged abdominal nodes were also demonstrated.
Following a palliative strategy, the patient underwent pancreatoduodenectomy (Whipple's procedure), due to intraoperative signs of pancreatic involvement, and post-operative chemo and radiotherapy, as per consensus of familiar and multidisciplinary team engaged in the patient's care. The patient was discharged and indicated significant improvement from initial symptoms at a follow-up consultation two months after surgery, but did not attend subsequent outpatient clinic.

\section{Discussion}

Coloduodenal fistulae are classified, accordingly to its cause, in benign or malignant. Crohn's disease and duodenal ulcers have been reported as the most common benign causes. Iatrogenic fistulas, penetrating injuries, foreign bodies and other inflammatory conditions, such as ulcerative colitis, colonic diverticulitis, radiation enteritis, and other abdominal infectious processes have also been described as causes of coloduodenal fistulae [1-4]. Malignant fistulae are usually secondary to colonic tumours, mainly those located at the hepatic flexure or the transverse colon [2]. However, primary lesions from stomach, small bowel, pancreas, gallbladder and lymphoma have already been cited as cause [4]. Metastases of oesophageal cancer progressing to coloduodenal fistulae have also been reported [5].

Gastrointestinal bacterial overgrowth caused by the upward migration of the colonic content is cited among the causes of the most frequent symptoms, which include diarrhoea, abdominal pain and nausea. Occasionally, fecaloid vomiting may occur. The direct leakage of pancreatic and biliary secretions into the colonic lumen through the fistula is associated with the development of secretory diarrhoea, metabolic acidosis and chronic malabsorption, reflecting a bypass mechanism [1]. If not treated, necessitans cutaneous fistulisation can occur.

Barium enema has an accuracy of approximately $90 \%$ and shows better sensitivity than barium meal or gastrografin swallow [6], though it was not performed in the reported case due to the low 


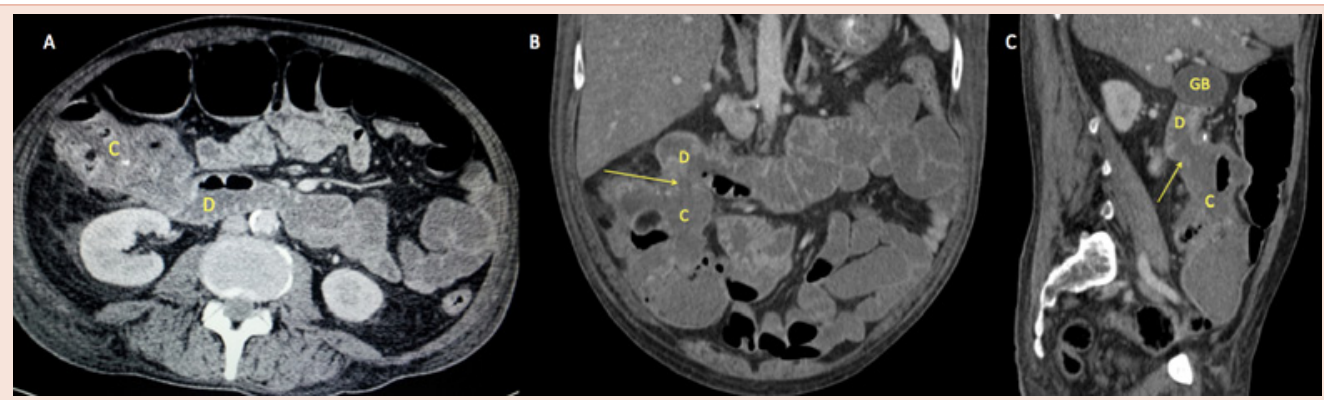

Figure 1: A-axial, B-coronal and C-sagittal views, $\mathrm{C}=$ colon / $\mathrm{D}=$ duodenum and $\mathrm{GB}=$ gallbladder.

clinical suspicion for enteric fistulae. As such, the condition may be incidentally diagnosed by MDCT or magnetic resonance imaging (MRI) during scanning for unexplained abdominal pain or palpable masses. Multiplanar imaging reconstructions in coronal, sagittal, oblique and curved views (MPR) are increasing the detectability of the fistulous tract. However, in clearly suspected cases, such as those presenting with fecaloid vomiting, barium enema can easily demonstrate the shunt between the colon and the duodenum and figures as the method of choice. On the other hand, MDCT and MRI have the advantage of providing the imaging staging, when the cause is a malignant process. Notwithstanding this, because of the condition 's low prevalence, there is a lack of cost-effectiveness studies outlining unequivocal benefits of cross-sectional modalities. Direct visualisations of the fistulous tract by upper or lower endoscopy are also feasible approaches, showing variable results, depending on the experience of the operator [7]. It is noteworthy that barium enema study should be performed whenever possible (i.e., in nonemergency scenarios) and available, as it is still the method of choice for the assessment of coloduodenal fistulae due to its high diagnostic sensitivity.

The management of an enteroenteric fistula may differ, depending on the affected segments of the duodenum and colon and the clinical status of the patient [3]. At some point, most cases will demand surgical intervention $[7,8]$. En bloc tumoral resection with microscopic negative margins is usually recommended, as well as simultaneous resection of liver implants when evidenced [7], though the presented therapy has been previously reported as successful when invasion of adjacent organs exist [9]. Finally, more conservative treatments may be reasonable for patients with a poor clinical status or bowel inflammatory diseases.

In summary, although coloduodenal fistulae are uncommon, one should be alerted to this possibility. In addition to barium enema, cross-sectional abdominal imaging studies, especially MDCT and MRI, should always be assessed with MPR views, which may make it easier to suspect or to confirm the presence of a fistulous tract. This is particularly important, in patients with advanced colon cancer in the hepatic flexure or transverse colon, with infiltration of the adjacent fat and with persistent diarrhoea or fecaloid vomit.

\section{References}

1. Ng CK, Cheung YS, Wong CH, Li KW (2009) Coloduodenal fistula: a rare complication of right-sided diverticulitis. Singapore Med J 50: 220-222.

2. Majeed TA, Gaurav A, Shilpa D, Preeti J, Sanjay S, et al. (2011) Malignant coloduodenal fistulas-review of literature and case report. Indian J SurgOncol 2: $205-209$

3. Pronisceva V, Bagla N, Lakshmaiah S, Sharp E (2012) Rare presentation of primary coloduodenal fistula. JRSM Short Rep 3: 29.

4. Soulsby R, Leung E, Williams N (2006) Malignantcolo-duodenal fistula; case report and review of the literature. World $\mathrm{J}$ SurgOncol 4: 86 .

5. Reissman P, Steinhagen RM, Enright PF (1992) Duodeno-colic fistula: an unusual presentation of esophageal squamous cell carcinoma. Mt Sinai J 59: $75-78$.

6. Pichney LS, Fantry GT, Graham SM (1992) Gastrocolic and duodenocolic fistulas in Crohn's disease. J ClinGastroenterol 15: 205-211.

7. Kamath AS, Iqbal CW, Pham TH, WolffBG, Chua HK, et al. (2011) Management and outcomes of primary coloduodenal fistulas. J GastrointestSurg 15: 17061711.

8. El-Hajj II, Abdul-Baki H, El-Zahabi LM, Barada KA (2007) Primary coloduodenal fistula in Crohn's disease. Dig Dis Sci 52: 59-63.

9. Yoshimi F, Asato Y, Kuroki Y, Shioyama Y, Hori M, et al. (1999) Pancreatoduodenectomy for locally advanced or recurrent colon cancer: report of two cases. Surg Today 29: 906-910. 